\title{
Protocolo diagnóstico del cáncer de origen desconocido. Revisión de 157 casos
}

\author{
A. ANTUÑA EGOCHEAGA, Mª . L. LÓPEZ GONZÁLEZ, J. LOBO FERNÁNDEZ*, \\ J. FERNÁNDEZ BUSTAMANTE**, J. MORIS DE LA TASSA**, A. CUETO ESPINAR
}

Departamento de Medicina Preventiva y Salud Pública. Facultad de Medicina. Universidad de Oviedo. *Servicios de Radiología y **Medicina Interna. Hospital de Cabueñes. Gijón

\author{
UNKNOWN PRIMARY CANCER: DIAGNOSTIC PROTOCOL \\ REVISION OF 157 CASES
}

\section{RESUMEN}

Objetivo: La toma de decisiones diagnósticas en el Cáncer de Origen Desconocido (COD) está sujeta a gran variabilidad. La aplicación de un protocolo diagnóstico basado en la evidencia científica actual, podría evitarla. Se describen las características epidemiológicas del COD en Asturias y se evalúa la adecuación de las pruebas diagnósticas realizadas al protocolo teórico.

Método: Estudio descriptivo retrospectivo (1992-96).

Resultados: En el estudio piloto se incluyeron 157 casos: 59\% varones, con edad media global de 67 años. La forma de presentación más frecuente fue hepatomegalia $(29 \%)$ y la histología: adenocarcinoma (51\%). El diagnóstico del primario se hizo en el 22\%, localizándose en pulmón en el 31\%; se hizo necropsia sólo en el 4,8\% de los fallecidos. La mediana de la supervivencia fue de 13 semanas, significativamente superior para el carcinoma escamoso, sin relación con el éxito en el diagnóstico del primario. La media de estudios innecesarios por paciente fue de: 8 analíticas, 3 técnicas de imagen y 0,6 técnicas agresivas. El desvío respecto del protocolo teórico fue superior al $25 \%$ en un $47 \%$ de los casos.

Conclusiones: La aplicación de un protocolo diagnóstico en el estudio del COD evitaría pruebas diagnósticas innecesarias, con el consiguiente ahorro de molestias para el paciente y de costes sanitarios.

PALABRAS CLAVE: Cáncer de origen desconocido. Protocolo diagnóstico. Variabilidad

\begin{abstract}
Objective: Decision-making to diagnose an Unknown Primary Can cer $(U P C)$ is subject to great variability. It is possible to design a stan dard procedure using the scientific literature, wich seems to be able to avoid this variability. We describe the characteristics of the UPC in Asturias and measure the degree of adaptation of the diagnostic deci sions to the theoretic standard procedure.

Method: Descriptive retrospective study (1992-96)

Results: A pilot study included 157 cases: mean age 67 years and $59 \%$ male. The presentation form most frecuent was hepatomegaly (29\%) and histology: adenocarcinoma (51\%). The diagnosis of the pri mary was possible in 22\%: lung (31\%). Median survival: 13 weeks, hig her for squamous carcinoma, but independent of the diagnosis of the primary tumour. Comparing with the recommended protocol, the average of unnecessary diagnostic techniques per patient was: 8 analyses, 3 ima ge studies and 0,6 unnecesary aggresive techniques.

Conclusions: The great variability in decision-making for diagno sing advises the application of a diagnostic protocol that would avoid innecesary damage for the patient and sanitary costs.
\end{abstract}

KEY WORDS: Unknown primary cancer. Variability. Diagnostic pro cedure.

Antuña Egocheaga A, López González Má L, Lobo Fernández J, Fernández Bustamante J, Moris de la Tassa J, Cueto Espinar A. Protocolo diagnóstico del cáncer de origen desconocido. Revisión de 157 casos. An Med Interna (Madrid) 2002; 19: 405-408.

\section{INTRODUCCIÓN}

El COD se define como una lesión maligna confirmada histológicamente, -incompatible con un tumor primario en la zona de la biopsia-, cuyo origen no se ha identificado, a pesar de una anamnesis cuidadosa, un examen físico completo, estudios básicos de laboratorio, radiografía simple de tórax y estudios adicionales dirigidos por los hallazgos iniciales $(1,2)$.

En las decisiones diagnósticas y terapéuticas de esta enfermedad metastásica se detecta gran variabilidad. Sin embargo, estudios recientes demuestran que es posible protocolizar las exploraciones diagnósticas con el fin de identificar los tumores primarios subsidiarios de tratamiento específico, y prevenir complicaciones locales inminentes, en un intento de mejorar la calidad de vida del enfermo (2-6).

Se han revisado los casos diagnosticados de COD en Asturias en el quinquenio 1992-96 con el objetivo de describir sus características epidemiológicas, las decisiones diagnósticas tomadas en estos pacientes y el grado de adecuación de estas decisiones a un protocolo teórico.

Trabajo aceptado: 23 de julio de 1999

Correspondencia: Aurora Antuña Egocheaga. Camino de la Cantera nº 632. Cimadevilla. Cabueñes. 33394 Gijón. Asturias. e-mail: casayoya @ hotmail.com 


\section{MATERIAL Y MÉTODOS}

Se han incluido todos los pacientes diagnosticados de COD en los hospitales de nuestra comunidad autónoma durante el período 1992-96, seleccionando una muestra de 157 casos para un estudio piloto. En la definición de caso se ha asumido la establecida en la literatura científica: "Neoplasia maligna histológicamente demostrada cuyo tumor primario no se evidencia tras historia clínica, exploración física completa, estudios básicos de laboratorio (hemograma, bioquímica de sangre y orina, electroforesis de suero y test de sangre oculta en heces) y radiografía de tórax, añadiendo sólo aquellos estudios adicionales que sean sugeridos por anormalidades detectadas en los estudios iniciales“ $(1,2)$. Criterios de inclusión: evidencia histológica de malignidad, incompatible con un tumor primario en la zona biopsiada y ausencia de tumor primario identificado según la definición de caso. Cri terios de exclusión: evidencia clínico-radiológica de maligni$\mathrm{dad}$, sin demostración histológica.

Diseño de la investigación: estudio descriptivo longitudinal, retrospectivo (92-96). Variables a estudio: demográficas, tiempo de estancia hospitalaria (días), datos clínicos, histología y localización de la metástasis. Las exploraciones diagnósticas se dividieron en: analítica, marcadores tumorales, estudios de imagen, técnicas agresivas (endoscopias, biopsias adicionales o cirugía) e inmunohistoquímica de la metástasis. Diagnóstico del primario: tiempo transcurrido hasta el diagnóstico y características del mismo (tipo de tumor, in vivo o post-mortem, probable o definitivo, confirmación necrópsica). Tipo de tratamiento, evolución y análisis de la supervivencia. Nivel de ajuste al protocolo.

El protocolo teórico utilizado se elaboró en base a la evidencia científica disponible tras una revisión exhaustiva y actualizada (2-5) (Tabla I).

Recogida de datos: las fuentes fueron el Registro de tumores del Principado de Asturias y el de los hospitales que disponían de ellos. Un único investigador hizo la recogida de datos en una hoja informatizada común para todos los hospitales. Análisis de los datos: se procesaron con el programa dBASE IV y se analizaron con el programa SPSS para Windows, realizando estadística descriptiva de cada una de las variables y estadística analítica (t de Student y análisis de varianza para la comparación de medias y Chi-cuadrado para la comparación de proporciones).

\section{RESULTADOS}

Se incluyeron 157 casos; el 59\% fueron varones. La edad media fue de $67 \pm 11$ años (35-94), significativamente superior en la mujeres $(\mathrm{p}<0,01)$. La distribución por servicios fue la siguiente: M. Interna (47\%), Digestivo (12\%), Cirugía General (8\%), ORL (8\%) y otros (25\%) (Fig. 1). En el $44 \%$ se recogía un síndrome general, clínica digestiva en el 40\%, cardiorrespiratoria en $16 \%$, dolores óseos en $12 \%$ y en el $20 \%$ se había detectado una adenopatía, masa o bultoma; en la mitad de los casos coexistía más de un síntoma, con un tiempo medio de evolución de 13 semanas (intervalo 2-99) (Fig. 2). La exploración física reveló: hepatomegalia o nódulos hepáticos por ecografía (29\%), ascitis (22\%), adenopatías cervicales (14\%), derrame pleural (11\%) y metástasis óseas $(8 \%)$
TABLA I

PROTOCOLO DIAGNÓSTICO EN EL ESTUDIO DEL COD

\begin{tabular}{|c|c|c|}
\hline Tipo histológico & Estudio clínico & Técnicas histológicas \\
\hline $\begin{array}{l}\text { Adenocarcinoma } \\
\text { Bien } \\
\text {-moderadamente } \\
\text { diferenciado }\end{array}$ & $\begin{array}{l}\text { Tac abdomen } \\
\text { Adenopatia axilar } \\
\text { en mujer: M amografia } \\
\text { Ascitis en mujer: CA } 125 \\
\text { Varón: PSA } \\
\text { Adenop. cervical: } \\
\text { Varón (si PSA negativo) } \\
\text { M ujer }\end{array}$ & $\begin{array}{l}\text { Receptores estrógeno } \\
\text {-progesterona (M) } \\
\text { PSA tisular (h) } \\
\text { Tiroglobulina }\end{array}$ \\
\hline $\begin{array}{l}\text { Carcinoma escamos } \\
\text { Cervical }\end{array}$ & $\begin{array}{l}\text { So } \\
\text { Adenop.cervical: } \\
\text { TAC/RNM cuello } \\
\text { ORL endoscopia } \\
\text { (TAC tórax } \\
\text { +/ - broncoscopia) }\end{array}$ & Ninguun \\
\hline Inguinal & $\begin{array}{l}\text { Adenop.inguinal: } \\
\text { ANU-rectoscopia } \\
\text { Ginecología }\end{array}$ & \\
\hline $\begin{array}{l}\text { Carcinoma } \\
\text { indiferenciado }\end{array}$ & $\begin{array}{l}\text { TAC tórax/abdomen } \\
\text { AFP-BHCG-NSE } \\
\text { Lesión ósea: } \\
\text { Proteinuria Bence-Jones }\end{array}$ & $\begin{array}{l}\text { Inmunoperoxidasa } \\
\text { (M. electronica) } \\
\text { (Cariotipo) }\end{array}$ \\
\hline
\end{tabular}

H = Hombre; M = M ujer. (Tomado de Hainsworth y Greco (1993); Dauggard, 1994; Mayordomo, 1994 y NCCN Practice Guidelines, 1998).

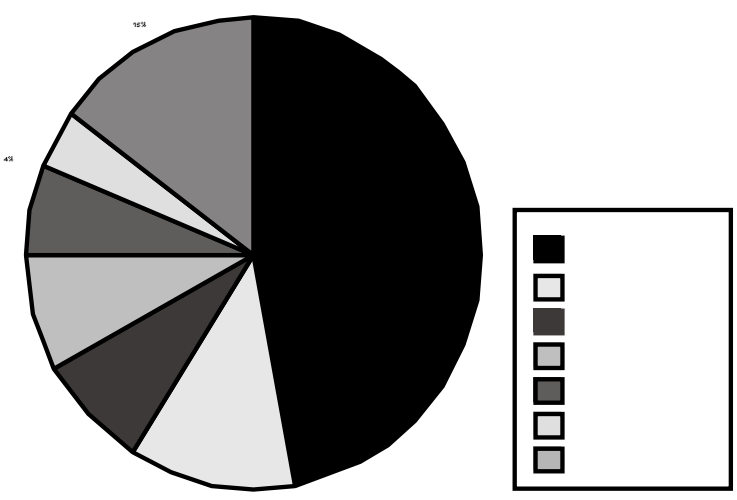

Fig. 1. Procedencia de los casos de COD por servicios.

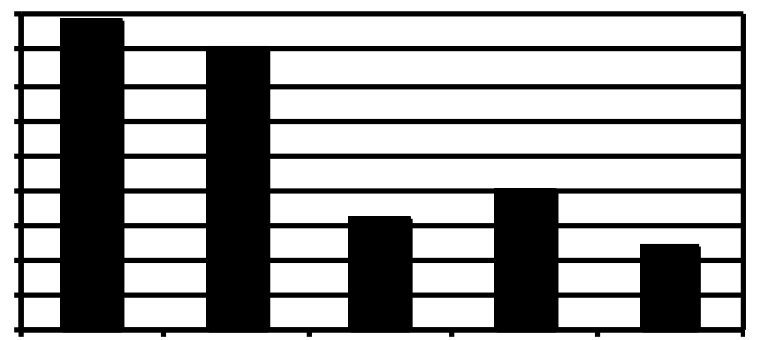

Fig. 2. Manifestaciones clínicas iniciales del COD. 
El $95 \%$ de los pacientes fue estudiado mediante ingreso hospitalario con una estancia media de 24 dias (3-107). El diagnóstico histológico de la metástasis incluyó: adenocarcinoma $(51 \%)$, carcinoma indiferenciado $(31 \%)$, carcinoma escamoso $(11 \%)$, neoplasia indiferenciada $(6 \%)$ y sarcoma (<1\%) (Fig. 3). Las muestras iniciales se obtuvieron por punción aspiración (PAAF) en el 50\%, 20\% biopsia, 18\% citología y ambas en el $11 \%$ (PAAF o citología y biopsia). Los tejidos en los que se diagnosticó el COD fueron : hígado (28\%), ganglio linfático $(21 \%)$, peritoneo $(19 \%)$, pleura $(10 \%)$, hueso $(6 \%)$ y otros $(16 \%)$

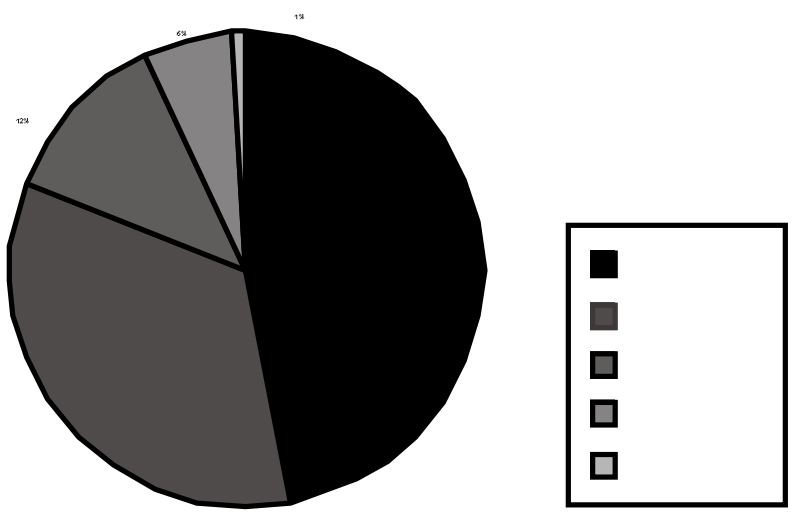

Fig. 3. Histología inicial del COD.

El diagnóstico del tumor primario fue posible en vida del paciente en 35 casos (22\%) y se localizó en: pulmón (31\%), riñón $(14 \%)$, páncreas $(12 \%)$, ovario $(12 \%)$, estómago $(6 \%)$, próstata $(6 \%)$, tiroides $(6 \%)$, y suprarrenal, melanoma, testículo, carcinoide y tumor papilar extraovárico en menos del $<1 \%$ cada uno (Fig. 4). La confirmación histológica del tumor primario sólo se consiguió en 11 casos (7\%). Entre los métodos diagnósticos de primario probable destacan la TAC abdominal (34\%) y torácica (16\%), ecografía abdominal (16\%) y el estudio de la metástasis mediante técnicas de inmunohistoquímica en 9\% (Tabla II). El 75\% de los diagnósticos se consiguió durante el primer ingreso y el resto durante el seguimiento, siendo el tiempo medio desde la detección del COD hasta el diagnóstico de 14 días (0-497 días). Sólo en 5 de los pacientes fallecidos $(4,8 \%)$ se realizó necropsia, siendo diagnóstica en todos los casos: 3 tumores de páncreas, 1 de pulmón y 1 colangiocarcioma intrahepático .

Falleció el $66 \%$ de los pacientes, con una mediana de la supervivencia de 13 semanas. El porcentaje de supervivencia acumulada a 1,3 y 5 años fue del $23 \%, 11,9 \%$ y $10,5 \%$ respectivamente. La supervivencia no se vio afectada por la edad, sexo o el éxito en el diagnóstico del primario $(p>0,05)$; en cambio sí fue significativamente superior en los carcinomas escamosos.

Se realizó una media de 8 análisis, 3 estudios de imagen y menos de 1 técnica agresiva innecesarios por paciente, si bien detectamos gran variabilidad en los diferentes casos. El nivel de ajuste al protocolo queda reflejado en la tabla III. Sólo en el 3\% de los casos el ajuste al protocolo fue perfecto, mientras que en el $47 \%$ el desvío fue superior al $25 \%$ y en el $11 \%$, al $50 \%$.

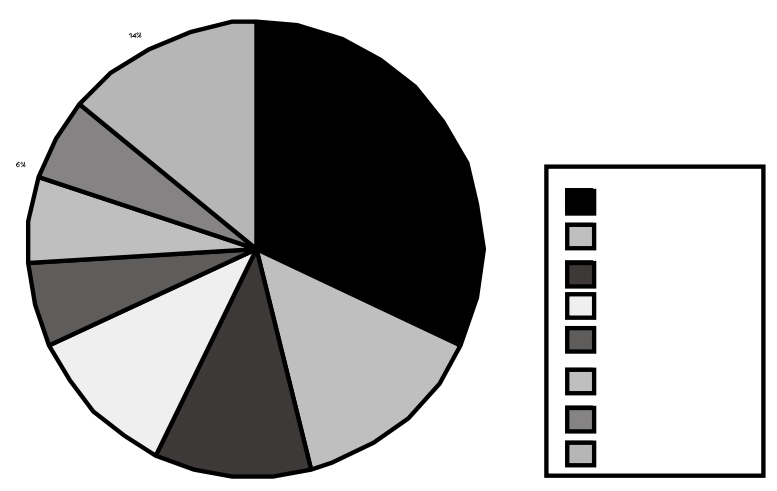

Fig. 4. Diagnóstico del primario "ante-mortem".

\begin{tabular}{lr}
\multicolumn{2}{c}{ TABLA II } \\
\multicolumn{2}{c}{ PRUEBAS DIAGNÓ STICAS DEL TUM O R PRIM ARIO } \\
\hline \multicolumn{2}{c}{$n^{0}$ casos (\%) } \\
\hline TAC abdominal & $11(31,5 \%)$ \\
TAC torácica & $5(14,4 \%)$ \\
Broncoscopia & $4(11,4 \%)$ \\
Inmunohistoquímica & $3(8,5 \%)$ \\
M arcador tumoral & $3(8,5 \%)$ \\
ECO de cuello + PAAF & $2(5,7 \%)$ \\
ECO abdominal & $2(5,7 \%)$ \\
Laparoscopia & $2(5,7 \%)$ \\
RX tórax de seguimiento & $2(5,7 \%)$ \\
Estudio gastroduodenal & $1(2,9 \%)$ \\
TO TAL & $35(100 \%)$ \\
\hline
\end{tabular}

TABLA III

AJUSTE AL PROTO COLO DIAGNÓSTICO

$n-\operatorname{cas} 0 \mathrm{~s}(\%)$

\begin{tabular}{lc}
\hline Ajuste del $100 \%$ & $4(3 \%)$ \\
Desvío $<25 \%$ & $62(39 \%)$ \\
Desvío $25-50 \%$ & $73(47 \%)$ \\
Desvío $>50 \%$ & $18(11 \%)$ \\
\hline
\end{tabular}

\section{DISCUSIÓN}

El porcentaje del COD en los registros hospitalarios de tumores oscila entre un $1,5 \%$ y un $10 \%$ del total $(5,7)$. Suele afectar por igual a ambos sexos, con una edad media de 57-59 años (8-12). En nuestra serie la edad media fue algo superior (67 años), y de forma significativa en las mujeres; no encontramos diferencias en el sexo.

La forma de presentación difiere notablemente en las series publicadas, con predominio de patología linfática en unos casos $(8,11)$ y ósea en otros $(12)$. En nuestros pacientes 
el hallazgo inicial más frecuente fue la afectación hepática, lo que coincide con los datos publicados por Shildt y cols. (13). Destacan los elevados porcentajes de carcinoma indiferenciado $(31 \%)$ y carcinoma escamoso (11\%), en relación a otras series $(3,8,12,14)$, si bien el adenocarcinoma continúa siendo la histología más frecuente, como recogen la mayoría de los autores.

La tomografía computarizada abdominal y torácica fueron las pruebas diagnósticas de mayor eficacia (34 y 16\% respectivamente), cifras que coinciden con otras publicaciones $(15,16)$. El valor de las técnicas de inmunohistoquímica para la detección del tumor primario a partir de muestras de la metástasis está ampliamente demostrado $(17,18)$, y en nuestra serie fue diagnóstica en el $9 \%$ de los casos.

Se consiguió el diagnóstico del primario en $22 \%$ de los casos, cifra coincidente con la publicada por otros autores $(8,10-12,14,19)$.

La mediana de la supervivencia oscila entre 3 y 6 meses y varía según el tipo histológico del tumor y la respuesta individual al tratamiento $(2,3,7,10,20)$. En nuestro estudio este valor fue algo inferior, si bien la supervivencia acumulada a 1,3 y 5 años fue coincidente con la de otras series (9). No encontramos relación significativa con la edad, el sexo o el éxito en la detección del primario, y sí con histología del COD (sobrevivían más los casos con carcinoma escamoso).

Se constató una utilización innecesaria de pruebas diagnósticas y gran variabilidad en la adecuación de las decisiones diagnósticas al protocolo teórico. Aunque no hemos encontrado referencias concretas a este aspecto en la literatura, cabe destacar su especial relevancia en esta situación de cáncer avanzado en la que el paciente suele presentar un deterioro físico importante, tiene pocas probabilidades de curación, y una supervivencia generalmente escasa que no siempre se ve afectada por la detección del tumor primario. En estas circunstancias las técnicas diagnósticas le van a provocar un sufrimiento añadido, y en el caso de las pruebas innecesarias, sin beneficio adicional. Los costes sanitarios derivados de ello serán por el mismo motivo inútiles y estériles.

La difusión y aplicación del protocolo propuesto, permitiría disminuir la enorme variabilidad en el estudio diagnóstico del COD y evitaría las consecuencias negativas antes mencionadas.

\section{Bibliografía}

1. Greco FA, Hainsworth JD. Cancer of unknown primary site. In: De Vita Jr. VT, Hellman S, Rosenberg SA, eds. Cancer: Principles and Practice of Oncology. 5th. ed. Philadelphia: Lippincott-Raven Publishers; 1997. p. $2423-43$

2. Hainsworth JD, Greco FA. Treatment of patients with cancer of an unknown primary site. N Engl J Med 1993; 329: 257-63.

3. Mayordomo JI, Guerra JM. Hacia una evaluación de los pacientes con cáncer de origen desconocido basada en un algoritmo histológico. Propuesta de un algoritmo. Med Clin (Barc) 1994; 102: 423-6.

4. Daugaard G. Unknown primary tumours. Cancer Treat Rev 1994; 20:119-147

5. NCCN Practice Guidelines for Occult Primary Tumors. Oncology 1998; 12: 226-309.

6. Hainsworth JD, Greco FA. Management of patients with cancer of unknown primary site. Oncology 2000; 14: 563-74

7. Altman E, Cadman E. An analysis of 1539 patients with cancer of unknown primary site. Cancer 1986; 57 : 120-4.

8. Le Chevalier T, Cvitkovic E, Caille P, Harvey J, Contesso G, Spielmann M, et al. Early metastatic cancer of unknown primary origin at presentation: a clinical study of 302 consecutive autopsied patients. Arch Intern Med 1988; 148: 2035-9.

9. Maiche AG. Cancer of unknown primary. A retrospective study based on 109 patients. Am J Clin Oncol 1993; 16: 26-9.

10. Abbruzzese JL , Abbruzzese MC, Hess KR, Raber MN, Lenzi R, Frost $\mathrm{Ph}$. Unknown primary carcinoma: natural history and prognostic factors in 657 consecutive patients. J Clin Oncol 1994; 12: 1272-80.

11. Stewart JF, Tattersall MHN, Woods RL, Fox RM. Unknown primary

adenocarcinoma: incide cinoma de origen desconocido. Estudio diagnóstico en 48 casos y su rentabilidad clínica. Med Clin (Barc) 1989; 92: 201-6.

13. Shildt RA, Kennedy PS, Chen TT, Athens JW, O’Bryan, Balcerzak SP. Management of patients with metastatic adenocarcinoma of unknown origin: a Southwest Oncology Group Study. Cancer Treat Rep 1983; 67: 77-9.

14. Ordóñez A, González M. Cáncer de origen desconocido: rentabilidad diagnóstica y terapéutica. Rev Clin Esp 1993; 193: 139-43.

15. Karsell PR, Sheddy PF, O'Connell MJ. Computed tomography in search of cancer of unknown origin. JAMA 1982; 248: 340-3.

16. McMillan JH, Levine E, Stephens RH. Computed tomography in the evaluation of metastatic adenocarcinoma from an unknown primary site: a retrospective study. Radiology 1982; 143: 143-6.

17. Mackay B., Ordonez NG. Pathological evaluation of neoplasms with unknown primary tumor site. Semin Oncol 1993; 20: 206-28.

18. Gatter KC, Alcock C, Heryet A, Maso DY. Clinical importance of analysing malignant tumours of uncertain origin with immunohistological techniques. Lancet 1985; 1: 1302-5.

19. Lenzi R, Hess KR, Abbruzzese MC, Raber MN, Ordoñez NG, Abbruzzese JL. Poorly differentiated carcinoma and poorly differentiated adenocarcinoma of unknown origin: favorable subsets of patients with unknown-primary carcinoma? J Clin Oncol 1997; 15: 2056-66.

20. Hess KR, Abbruzzese MC, Lenzi R, Raber MN, Abbruzzese JL. Classification and regression tree analysis of 1000 consecutive patients with unknown primary carcinoma. Clin Cancer Res 1999; 5: 3403-10. 